Check for updates

Cite this: Chem. Commun., 2019, 55, 12539

Received 30th August 2019,

Accepted 23rd September 2019

DOI: $10.1039 / c 9 c c 06729 a$

rsc.li/chemcomm

\section{A tunable detection range of ion-selective nano-optodes by controlling solvatochromic dye transducer lipophilicity $\dagger$}

\author{
Lu Wang and Eric Bakker (D) *
}

\begin{abstract}
A range of ionic solvatochromic dye (SD) transducers for use in ionselective emulsified optical sensors are introduced and characterized. They share the same chromophore group, (E)-4-(4-(dimethylamino)styryl)pyridinium, but vary in their lipophilicities by grafted alkyl or ethoxy groups. The calibration curve is found to shift by a total of 2.7 orders of magnitude with the lipophilicity of the SD.
\end{abstract}

Ion-selective optodes ${ }^{1-9}$ that function on the basis of ion-exchange typically use a lipophilic $\mathrm{pH}$ indicator ${ }^{10}$ (also called a chromoionophore) as an optical signal reporter. The target ions in the sample exchange with the hydrogen ions in the organic sensing phase, thereby deprotonating the chromoionophore and giving a change in absorbance or fluorescence. As hydrogen ions actively participate in the ion-exchange process, the sensors give an undesired cross response to sample $\mathrm{pH}$ changes. This was very difficult to overcome until the introduction of electrically charged solvatochromic dyes (SDs). ${ }^{11}$ The first proposed SDs ${ }^{12}$ were water-soluble and were therefore expelled into the bulk of the aqueous phase upon ion-exchange. This, however, contaminates the sample and gives signals that depend on the sample volume. To overcome these limitations, hydrophobic SDs have been introduced. ${ }^{13-16}$ This allows one to localize the ion-exchange process, rendering the ion optodes $\mathrm{pH}$-independent while avoiding dye leakage. Based on this characteristic, a reversible nylon-based optical sensor was reported. ${ }^{17}$ Recently, our group also showed that hydrophobic SD-based optical emulsion sensors fabricated without added surfactant exhibited improved selectivity owing to higher complex formation constants in that phase. ${ }^{18}$

Traditional chromoionophore based optical sensors give a response range that can be tuned by adjusting the $\mathrm{pH}$ of the sample. ${ }^{19}$ For monovalent target ions, a $1 \mathrm{pH}$-unit increase

University of Geneva, 30 Quai Ernest Ansermet, 1211 Geneva 4, Switzerland. E-mail: eric.backker@unige.ch; Tel: +41223796429

$\dagger$ Electronic supplementary information (ESI) available: Experimental details, photos of the probe, optical spectra of the SDs, calibration curves of the PVC/ DOS and DOS matrixed emulsion, lifetime calculation, partition coefficient calculation, synthesis procedure of SDs, and related NMR and mass spectrometry data. See DOI: 10.1039/c9cc06729a shifts the calibration curve by 1 order of magnitude to lower concentrations, while for divalent ions the shift is 2 orders of magnitude. ${ }^{20-22}$ Chromoionophore-based thermochromic optical sensors may also be effectively tuned by the loading of surfactant. $^{23}$

Replacing the chromoionophore by an SD does bring some drawbacks, as the lack of $\mathrm{pH}$ dependence now results in a calibration curve that is not easily tunable. ${ }^{12}$ In theory, the response range of such optical ion sensors depends on the ionexchange equilibrium. It depends on the complex formation constant between analyte and ionophore as well as the partition coefficient of the SD between the organic and aqueous phase. ${ }^{17}$ Complex formation constants are related to the structure of the ionophore and depend to some extent on the nature of the plasticizer, ${ }^{24}$ polymer and surfactant ${ }^{18,25}$ used in the sensing formulation. On the other hand, the partition coefficient of the SD may be adjusted by controlling its hydrophobic character. To study this, structurally different lipophilic SDs (SD-PEG4, SD-PEG2, SD-C1, SD-C4, SD-C18, see Scheme 1) were synthesized here and incorporated into $\mathrm{K}^{+}$-selective emulsion sensors to tune their response range.

Compared to the parent molecule SD-C1 (Scheme 1), ${ }^{17}$ SD-PEG4 and SD-PEG2 were obtained by structurally modifying the molecule with ethoxy groups, rendering them more hydrophilic. In contrast, SD-C4 and SD-C18 were designed to make the molecules more hydrophobic by adding alkyl chains $\left(-\mathrm{C}_{4} \mathrm{H}_{9}\right.$ or $\left.-\mathrm{C}_{18} \mathrm{H}_{37}\right)$ to the quaternary ammonium cation. The resulting lipophilicity is expected to increase in the order: SD-PEG4 < SD-PEG2 < SD-C1 < SD-C4 < SD-C18. The synthesized dyes were characterized by NMR and mass spectrometry (see the ESI $\dagger$ ). All five SDs share the same fluorophore and therefore exhibit similar excitation and emission wavelengths $(500 \mathrm{~nm} /$ $600 \mathrm{~nm}$, Fig. S1, ESI $\dagger$ ). The fluorescence quantum yields of these SDs (in EtOH) were found as 0.027, 0.031, 0.051, 0.052, and 0.070 , respectively, using fluorescein in $0.1 \mathrm{M}$ aqueous $\mathrm{NaOH}$ as in ref. 26 and 27. The empirically calculated parameters $\log P_{\mathrm{o} / \mathrm{w}}$ (logarithmic partition coefficient between the water and 1-octanol) for the five SDs are given in Table 1. If a neutral 


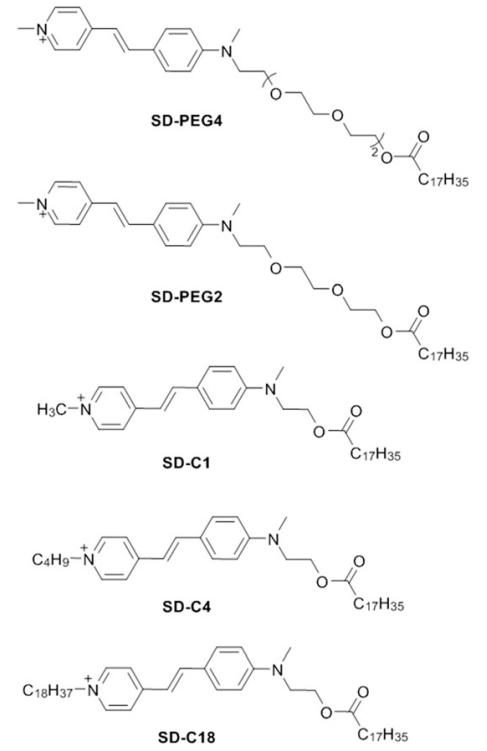

Scheme 1 The structures of the solvatochromic dyes. SD-PEG4 and SD-PEG2 have hydrophilic ethoxy groups grafted in the mother molecule SD-C1. SD-C4 and SD-C18 are gained by alkyl group change in the quaternary ammonium cation of SD-C1. The counter ion is iodide for each SD.

Table 1 Theoretical lipophilicity of different SDs (the whole molecule), expected calibration curve shift and practically gained shift value of the $\mathrm{K}^{+}$-selective PU-DOS emulsion

\begin{tabular}{|c|c|c|c|c|c|}
\hline & SD-PEG4 & SD-PEG2 & SD-C1 & SD-C4 & SD-C18 \\
\hline $\log P_{\mathrm{o} / \mathrm{w}}{ }^{a}$ & 4.44 & 4.63 & 4.80 & 5.65 & 8.14 \\
\hline $\log P$ & 3.95 & 4.10 & 4.24 & 4.92 & 6.91 \\
\hline Theoretical shift & \multirow{2}{*}{\multicolumn{2}{|c|}{0.15}} & 0.14 & 0.68 & 1.99 \\
\hline Practical shift & & & 0.4 & 0.6 & 1.0 \\
\hline
\end{tabular}

molecule exhibits a $\log P_{\mathrm{o} / \mathrm{w}}$ value higher than 8.56 , nanoparticles of $140 \mathrm{~nm}$ diameter may exhibit a loss of less than $10 \%$ during a $24 \mathrm{~h}$ period (see the ESI $\dagger$ for calculation). ${ }^{28,29}$ Structurally, each SD has a long chain functionality $\left(-\mathrm{CH}_{2} \mathrm{OCOC}_{17} \mathrm{H}_{35}\right)$ with a theoretical lipophilicity of $\log P_{\mathrm{o} / \mathrm{w}}=8.73$ (data from ALOGPS 2.1 program $^{30}$ ). In principle, therefore, all SDs exhibit the same strong linker to the organic phase that should prevent them from leaking out to the aqueous phase.

To standardize the results, the different SDs were incorporated into emulsified sensors for the detection of $\mathrm{K}^{+}$with valinomycin as ionophore. Emulsion particles were formed with the help of the SDs as amphiphilic molecules. ${ }^{18}$ The particles were made of an organic phase containing ion-exchanger (NaTFPB), $\mathrm{K}^{+}$ionophore I (valinomycin) and the SD of interest. Scheme 2 shows the mechanism of the ion-exchange process. The amphiphilic SD acts as a surfactant and stabilizes the organic nanodroplet phase ${ }^{18}$ When the ionic chromophore functional group of the $\mathrm{SD}$ exchanges with the target ion $\mathrm{K}^{+}$and moves from the organic to the aqueous phase, the solution polarity of the fluorophore group changes, resulting in fluorescence quenching. During this process, the SDs remain anchored to the organic phase with the help of the hydrophobic alkyl chain. ${ }^{13,17,18,31}$

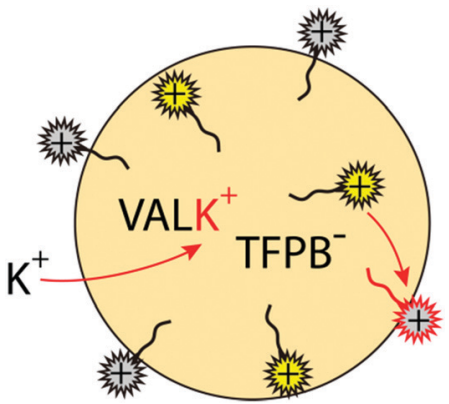

Scheme 2 Mechanism of the ion-exchange process.

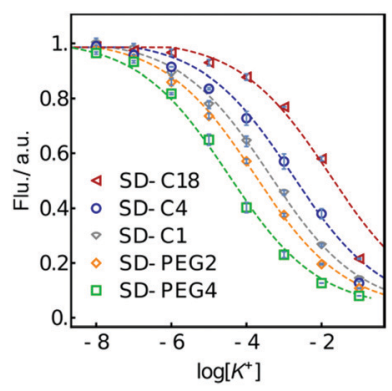

Fig. 1 Normalized fluorescence response of $\mathrm{K}^{+}$-selective emulsion sensors (PU-DOS) made from the indicated SDs. The dashed lines are to guide the eye.

The fluorescence response to $\mathrm{K}^{+}$for each formulation is shown in Fig. 1. The calibration curves are found to shift over 2.7 orders of magnitude to higher concentration with increasing lipophilicity of the SD. The trend is as expected, as sensors containing SDs with a hydrophilic PEG group exhibit a lower measuring range compared to SDs with a hydrophobic alkyl group because they exchange more easily with the potassium ion. One may expect the $\log P_{\mathrm{o} / \mathrm{w}}$ difference (Table 1) between two SDs to relate to the shift in the two corresponding calibration curves if all other parameters remain the same (see the ESI $\dagger$ for calculation).

The partition coefficient $P$ for the solvent system polymeric sensing membrane-water is known not to be strictly identical to the octanol-water partitioning coefficient, $P_{\mathrm{o} / \mathrm{w}}$, and the following relationship has been proposed for polymeric membranes to compare the two: ${ }^{32}$

$$
\log P=(0.8 \pm 0.1) \log P_{\mathrm{o} / \mathrm{w}}+(0.4 \pm 0.4)
$$

While the sensing material used here is not identical, this relationship is used here as a first approximation to estimate the calibration curve shift for the emulsion sensors. For example, the calibration curves of SD-C1 and SD-C4 based emulsions are expected to shift by 0.7 orders of magnitude, calculated from $(5.65-4.80) \times 0.8$. This corresponds well to the observed value of 0.6. The shift from SD-C4 to SD-C18 based $\mathrm{K}^{+}$-selective emulsions is smaller than expected, likely because the added alkyl chain does not fully extract into the aqueous phase upon expulsion but remains adsorbed onto the emulsion phase in analogy to the $\mathrm{C}_{17}$ anchor. Perhaps not very intuitively, calculations predict 
essentially no lipophilicity decrease when introducing a PEG spacer. Experimentally, a longer PEG chain shifts the measuring range to lower potassium concentrations by a total of 1.1 orders of magnitude, which may be explained by the electrostatic force between oxygen of the PEG and the hydrogen atom of the - $\mathrm{NH}-$ COO- group in the PU.

The calibration shift is also related to the matrix. Sensors prepared with PU-DOS have a larger shift and better repeatability than the sensors with PVC-DOS or DOS alone (see Fig. S2 and Table S1, ESI $\dagger$ ). Emulsion sensors containing SD-PEG4 give a higher measuring range than the one with SD-PEG2 when prepared with PVC-DOS or DOS, which runs counter to the lipophilicity difference. Generally, PU emulsions give a higher response range compared to PVC-DOS or DOS based emulsions. This is attributed to the urethane groups. The improved entrapment of SDs might be due to interactions between the cross-linked PU matrix and additional covalent bonds from amine functional groups of the carbamate structure (-NH-COO-). UV-vis and NMR spectra (Fig. S3, ESI $\dagger$ ) confirm that the PU used here contains benzene rings that might additionally retain aromatic dyes through $\pi-\pi$ interactions. In electrochemistry, it has been reported that the ion transfer peak position of cyclic voltammograms for membranes containing PU are at more positive potentials than the ones with PVC, ${ }^{33}$ suggesting stronger interactions with the matrix. This is consistent with the optical sensors studied here.

Table S2 (ESI $\dagger$ ) shows that the diameters of the different emulsion particles are all around $130 \mathrm{~nm}$ to $140 \mathrm{~nm}$ with $\zeta$-potentials at about -40 to $-50 \mathrm{mV}$. Standard deviations are calculated from three independent experiments.

Fig. 2 characterizes the selectivity of the emulsified sensors based on different SDs, with the corresponding selectivity coefficients from the horizontal distance between two calibration curves of the primary ion and interfering ion shown in Table $2 .^{34}$
Table 2 Selectivity coefficients of $\log K_{\mathrm{K}, \mathrm{Na}}^{\mathrm{opt}}$ and $\log K_{\mathrm{K}, \mathrm{TBA}}^{\mathrm{opt}}$ for different SD based $\mathrm{K}^{+}$-selective emulsion sensors

\begin{tabular}{llllll}
\hline & SD-PEG4 & SD-PEG2 & SD-C1 & SD-C4 & SD-C18 \\
\hline $\log K_{\mathrm{K}, \mathrm{Na}}^{\mathrm{opt}}$ & $\sim-5.3$ & -5.6 & -5.4 & -4.3 & -2.3 \\
$\log K_{\mathrm{K}, \mathrm{TBA}}^{\mathrm{ot}}$ & -1.5 & -1.5 & $\sim-0.5$ & $\sim-1.3$ & -1.5
\end{tabular}

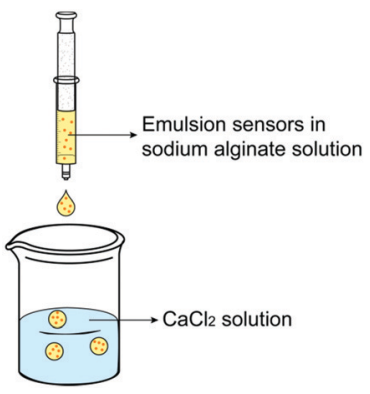

Scheme 3 Illustration of alginate gel encapsulation

Emulsion sensors with more lipophilic SDs give better selectivity over the interfering ion $\mathrm{Na}^{+}$. SD-C1 based $\mathrm{K}^{+}$-selective emulsion sensors show poor selectivity over $\mathrm{TBA}^{+}$. However, the other emulsified sensors exhibit similar logarithmic selectivity coefficients over $\mathrm{TBA}^{+}$, at about $\mathbf{- 1 . 5}$.

The stability of the $\mathrm{K}^{+}$-selective sensors was studied by encapsulating the emulsion in alginate gel spherical shells (Scheme 3). The pore size of the hydrated alginate gel has been reported to range from 6 to $17 \mathrm{~nm},{ }^{35,36}$ only allowing free molecules to pass through while blocking emulsion particles. Alginate microencapsulation is frequently used for cell culturing ${ }^{37}$ and drug delivery $^{38}$ owing to its biocompatibility and size selectivity. Here, five alginate particles (dia. ca. $4 \mathrm{~mm}$ ) were prepared for each kind of emulsion sensor, transferring them to $20 \mathrm{~mL}$ of
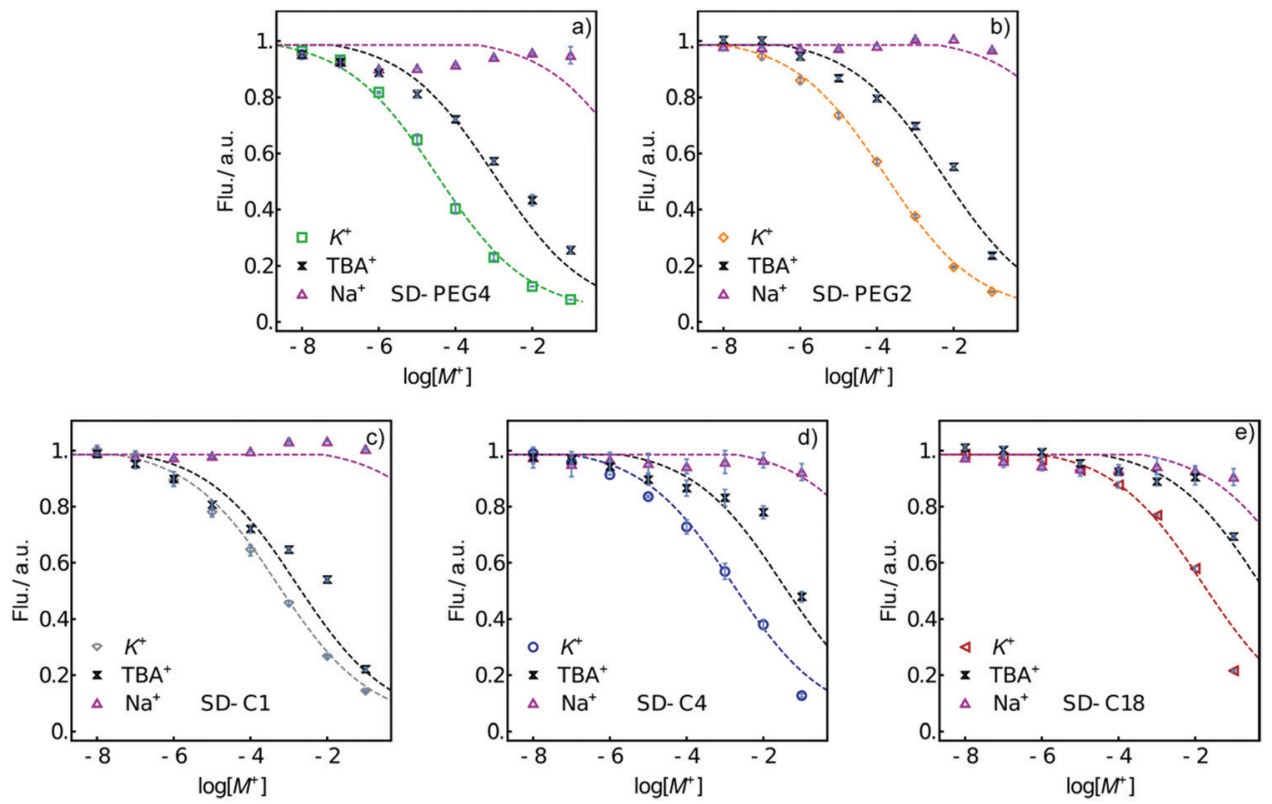

Fig. 2 Normalized fluorescence response of $\mathrm{K}^{+}$-selective emulsion sensor selectivity over $\mathrm{Na}^{+}$and $\mathrm{TBA}^{+}$with (a) SD-PEG4, (b) SD-PEG2, (c) SD-C1, (d) SD-C4, and (e) SD-C18. Error bars: standard deviations from triplicates. The dashed curves are drawn to guide the eye. 


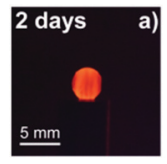

$1.04 \pm 0.05$

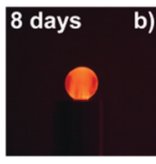

$1.14 \pm 0.10$

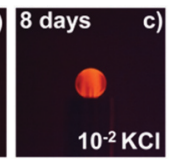

$0.32 \pm 0.05$

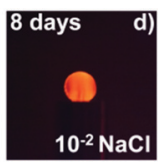

$0.87 \pm 0.04$
Fig. 3 Fluorescence microscope images of alginate particles containing SD-C1 based emulsion sensors stored in Milli-Q water for (a) 2 days and (b) 8 days. 8 day old alginate particle in contact with (c) $10^{-2} \mathrm{M} \mathrm{KCl}$ and (d) $10^{-2} \mathrm{M} \mathrm{NaCl}$ for one hour. Optical filter used: LP590. The number below each image is the average fluorescence intensity of the 5 observed particles.

Milli-Q water and keeping them in the dark at room temperature. Fluorescence recorded by the fluorescence probe is shown in Fig. S4 (ESI $\dagger$ ). All different formulations of alginate particles exhibited similar behavior, so only the images and fluorescence intensity values of the alginate particles containing SD-C1 based emulsion are given here. Fig. $3 \mathrm{a}$ and $\mathrm{b}$ show that the fluorescence intensity is stable for at least 8 days. The alginate particles were immersed after the 8 day period in $20 \mathrm{~mL}$ of $10^{-2} \mathrm{M} \mathrm{KCl}$ or $10^{-2} \mathrm{M} \mathrm{NaCl}$ solution for $1 \mathrm{~h}$, see Fig. $3 \mathrm{c}$ and d. The response and selectivity are still adequate after 8 days. The responses are slightly different compared to the data in Fig. 2c, likely because of the presence of residual $\mathrm{Na}^{+}$and $\mathrm{Ca}^{2+}$ from the alginate preparation that might contribute to the response. This preliminary experiment suggests that emulsion particle sensors may be encapsulated into alginate shells of micrometer size for application in bioanalysis.

In this work, we successfully developed ion-selective sensors with a tunable response range by structurally controlling the lipophilicity of the SD molecules. We synthesized SDs of different lipophilicity by modifying the molecule with alkyl or PEG side chains. Emulsion sensors were prepared with these SDs and other sensor components such as ion-exchanger, ionophore and plasticizer, with or without added polymer (PU or PVC). The calibration curve was found to shift to higher concentration with increasing lipophilicity of the SD. We also found that the PU polymer-based emulsion sensors gave a larger calibration curve shift and better repeatability than with the other two matrices. The selectivity of the $\mathrm{K}^{+}$-selective sensors over $\mathrm{Na}^{+}$was found to decrease with increasing lipophilicity of the SDs. Additionally, the sensors (PU-DOS) gave good long-term stability. No obvious dye leakage was found and they remained functional after 8 days. Encapsulated with the alginate gel, these ion-selective emulsion sensors are potentially attractive for on-site, bioanalytical and imaging applications exhibiting a tunable measurement range.

The authors thank the Swiss National Science Foundation (SNF) and the University of Geneva for financial support.

\section{Conflicts of interest}

There are no conflicts to declare.

\section{Notes and references}

1 P. Buhlmann, E. Pretsch and E. Bakker, Chem. Rev., 1998, 98, 1593.

2 K. Seiler and W. Simon, Sens. Actuators, B, 1992, 6, 295.

3 B. Awqatty, S. Samaddar, K. J. Cash, H. A. Clark and J. M. Dubach, Analyst, 2014, 139, 5230.

4 J. M. Dubach, D. I. Harjes and H. A. Clark, Nano Lett., 2007, 7, 1827.

5 G. X. Rong, E. H. Kim, K. E. Poskanzer and H. A. Clark, Sci. Rep., 2017, 7, 1.

6 C. Y. Yang, Y. Qin, D. C. Jiang and H. Y. Chen, ACS Appl. Mater. Interfaces, 2016, 8, 19892.

7 M. D. Kim, S. A. Dergunov, E. Lindner and E. Pinkhassik, Anal. Chem., 2012, 84, 2695.

8 S. A. Dergunov, B. Miksa, B. Ganus, E. Lindner and E. Pinkhassik, Chem. Commun., 2010, 46, 1485.

9 X. D. Wang, R. J. Meier and O. S. Wolfbeis, Angew. Chem., Int. Ed., 2013, 52, 406.

10 J. Y. Han and K. Burgess, Chem. Rev., 2010, 110, 2709.

11 C. Reichardt, Chem. Rev., 1994, 94, 2319.

12 X. Xie, A. Gutierrez, V. Trofimov, I. Szilagyi, T. Soldati and E. Bakker, Anal. Chem., 2015, 87, 9954.

13 X. Xie, I. Szilagyi, J. Zhai, L. Wang and E. Bakker, ACS Sens., 2016, $1,516$.

14 C. Krause, T. Werner, C. Huber and O. S. Wolfbeis, Anal. Chem., 1999, 71, 5304.

15 O. S. Wolfbeis, Sens. Actuators, B, 1995, 29, 140.

16 I. Murkovic, A. Lobnik, G. J. Mohr and O. S. Wolfbeis, Anal. Chim. Acta, 1996, 334, 125.

17 L. Wang, X. Xie, J. Zhai and E. Bakker, Chem. Commun., 2016, 52, 14254.

18 L. Wang, S. Sadler, T. Cao, X. Xie, J. M. Von Filseck and E. Bakker, Anal. Chem., 2019, 91, 8973.

19 M. Lerchi, E. Bakker, B. Rusterholz and W. Simon, Anal. Chem., 1992, 64, 1534.

20 X. Xie and E. Bakker, Anal. Bioanal. Chem., 2015, 407, 3899.

21 X. Xie, J. Zhai and E. Bakker, Anal. Chem., 2014, 86, 2853.

22 M. Shortreed, E. Bakker and R. Kopelman, Anal. Chem., 1996, 68, 2656.

23 X. F. Du, C. Y. Zhu and X. Xie, Langmuir, 2017, 33, 5910.

24 Z. Szigeti, A. Malon, T. Vigassy, V. Csokai, A. Grun, K. Wygladacz, N. Ye, C. Xu, V. J. Chebny, I. Bitter, R. Rathore, E. Bakker and E. Pretsch, Anal. Chim. Acta, 2006, 572, 1.

25 X. Xie and E. Bakker, Anal. Chem., 2015, 87, 11587.

26 S. Fery-Forgues and D. Lavabre, J. Chem. Educ., 1999, 76, 1260.

27 A. T. R. Williams, S. A. Winfield and J. N. Miller, Analyst, 1983, 108, 1067.

28 O. Dinten, U. E. Spichiger, N. Chaniotakis, P. Gehrig, B. Rusterholz, W. E. Morf and W. Simon, Anal. Chem., 1991, 63, 596.

29 E. Bakker and E. Pretsch, Anal. Chim. Acta, 1995, 309, 7.

30 I. V. Tetko and V. Y. Tanchuk, J. Chem. Inf. Comput. Sci., 2002, 42, 1136.

31 L. Wang, X. Xie, T. Cao, J. Bosset and E. Bakker, Chem. - Eur. J., 2018, 24, 7921 .

32 U. Oesch and W. Simon, Anal. Chem., 1980, 52, 692.

33 M. Cuartero, G. A. Crespo and E. Bakker, Anal. Chem., 2016, 88, 5649. 34 D. C. Darrow, N. Engl. J. Med., 1950, 242, 1014.

35 J. Klein, J. Stock and K. D. Vorlop, Eur. J. Appl. Microbiol., 1983, 18, 86.

36 W. W. Stewart and H. E. Swaisgood, Enzyme Microb. Technol., 1993, $15,922$.

37 K. Alessandri, M. Feyeux, B. Gurchenkov, C. Delgado, A. Trushko, K. H. Krause, D. Vignjevic, P. Nassoy and A. Roux, Lab Chip, 2016, 16, 1593.

38 M. T. Conconi, E. DeCarlo, S. Vigolo, C. Grandi, G. Bandoli, N. Sicolo, G. Tamagno, P. P. Parnigotto and G. G. Nussdorfer, Horm. Metab. Res., 2003, 35, 402. 